\title{
Faintly compatible mappings and common fixed points
}

Ravindra K Bisht ${ }^{1}$ and Naseer Shahzad ${ }^{2 *}$

"Correspondence:

nshahzad@kau.edu.sa

${ }^{2}$ Department of Mathematics, King Abdulaziz University, P.O. Box 80203,

Jeddah, 21589, Saudi Arabia

Full list of author information is

available at the end of the article

\begin{abstract}
In this paper we introduce a generalization of the concept of compatible mappings, and using that condition, we obtain some new fixed point theorems under both contractive and noncontractive conditions, which may allow the existence of a common fixed point or the existence of multiple fixed or coincidence points. We also manifest that the new concept is a necessary condition for the existence of a common fixed point.
\end{abstract}

MSC: $47 \mathrm{H} 10 ; 54 \mathrm{H} 25$

Keywords: common fixed point; compatible maps; weak compatible maps; Lipschitz-type mappings

\section{Introduction and preliminaries}

The question of the existence of common fixed points of commuting continuous self-maps of a compact convex set had remained an open problem for a fairly long time. It was only in 1969 that Boyce [1] and Huneke [2] independently proved that there exist two continuous commuting self-maps of the unit interval $[0,1]$ without a common fixed point. Thus, the study of common fixed points of a pair of self-mappings satisfying contractive-type conditions becomes interesting in view of the fact that even commuting continuous mappings on such nicely behaved entities as compact convex sets may fail to have a common fixed point. When we extend such studies to the class of noncommuting contractive-type mapping pair, it becomes still more interesting.

In 1982, Sessa [3] obtained the first weaker version of commutativity by introducing the notion of weak commutativity. This concept was further generalized by Jungck when he defined the concept of compatible mappings [4]. Extending weak commuting mappings, Pant [5] introduced the notion of R-weak commutativity. In 1996 Jungck again generalized the notion of compatible mappings by introducing weakly compatible mappings [4]. In 2008, Al-Thagafi and Shahzad [6] weakened the notion of nontrivial weakly compatible maps by introducing a new notion of occasionally weakly compatible (in short, owc) maps. In the recent work, Pant and Pant [7] redefined the notion of occasionally weakly compatible mappings by conditional commutativity. Over the past few years, generalizations of weakly compatible mappings and owc have been extensively used to study common fixed points of contractive mappings. In a recent note Alghamdi et al. ([8], see also [9] and references therein) have shown that many recent results which employ several weaker noncommuting notions are not real generalizations of previously existing results on weakly 
compatible mappings. They have also shown that many of the generalized commutativity conditions including owc fall in the subclass of weak compatibility in the setting of a unique common fixed point (or a unique point of coincidence). Those new classes of noncommuting notions are interesting but contractive conditions do not provide an ideal setting for the application of these concepts. For proper applications of these notions, one should look to mappings satisfying nonexpansive conditions, Lipschitz-type conditions or some other general conditions [10].

In 2011, Haghi et al. [11] proved a powerful lemma and showed that some coincidence and common fixed point generalizations in fixed point theory are merely consequences of the corresponding fixed point theorems existing in the literature (for more details, see [11]).

Fixed point theorems are statements containing sufficient conditions that ensure the existence of a fixed point. Common fixed point theorems invariably require a commutativity condition, a condition on the ranges of the mappings, some continuity condition, and a contractive or possibly a Lipschitz-type condition and every significant fixed point theorem attempts to weaken or obtain a necessary version of one or more of these conditions (see, for instance, [12-16]).

Before proceeding further, we recall some relevant concepts.

Definition 1.1 A pair of self-maps $(A, S)$ of a metric space $(X, d)$ is said to be

(i) compatible [17] if and only if $\lim _{n \rightarrow \infty} d\left(A\left(S\left(y_{n}\right)\right), S\left(A\left(y_{n}\right)\right)\right)=0$, whenever $\left\{y_{n}\right\}$ is a sequence in $X$ such that $\lim _{n \rightarrow \infty} A\left(y_{n}\right)=\lim _{n \rightarrow \infty} S\left(y_{n}\right)=t$ for some $t$ in $X$;

(ii) noncompatible if there exists a sequence $\left\{y_{n}\right\}$ in $X$ such that $\lim _{n \rightarrow \infty} A\left(y_{n}\right)=\lim _{n \rightarrow \infty} S\left(y_{n}\right)=t$ for some $t$ in $X$, but $\lim _{n \rightarrow \infty} d\left(A\left(S\left(y_{n}\right)\right), S\left(A\left(y_{n}\right)\right)\right)$ is either non-zero or non-existent;

(iii) weakly compatible [4] if the pair commutes on the set of coincidence points (a point $x \in X$ is called a coincidence point of the pair $(A, S)$ if $A(x)=S(x)$ ), i.e., $A(S(x))=S(A(x))$ whenever $A(x)=S(x)$ for some $x \in X$;

(iv) occasionally weakly compatible [6] if there exists a coincidence point $x$ in $X$ such that $A(x)=S(x)$ implies $A(S(x))=S(A(x))$;

(v) conditionally commuting [7] if the pair commutes on a nonempty subset of the set of coincidence points whenever the set of coincidences is nonempty;

(vi) subcompatible [18] if there exists a sequence $\left\{y_{n}\right\}$ in $X$ such that $\lim _{n \rightarrow \infty} A\left(y_{n}\right)=\lim _{n \rightarrow \infty} S\left(y_{n}\right)=t \in x$ and $\lim _{n \rightarrow \infty} d\left(A\left(S\left(y_{n}\right)\right), S\left(A\left(y_{n}\right)\right)\right)=0$;

(vii) conditionally compatible [10] if and only if whenever the set of sequences $\left\{y_{n}\right\}$ satisfying $\lim _{n \rightarrow \infty} A\left(y_{n}\right)=\lim _{n \rightarrow \infty} S\left(y_{n}\right)$ is nonempty, there exists a sequence $\left\{z_{n}\right\}$ such that $\lim _{n \rightarrow \infty} A\left(z_{n}\right)=\lim _{n \rightarrow \infty} S\left(z_{n}\right)=t$ and $\lim _{n \rightarrow \infty} d\left(A\left(S\left(z_{n}\right)\right), S\left(A\left(z_{n}\right)\right)\right)=0$.

It may be observed that compatibility is independent of the notion of conditional compatibility, and in the setting of a unique common fixed point (or unique point of coincidence), conditional compatibility does not reduce to the class of compatibility. The following examples illustrate these facts.

Example 1.1 Let $X=[1, \infty)$ with the Euclidean metric $d$. Define mappings $A, S: X \rightarrow X$ by

$$
A(x)=x \quad \forall x \quad \text { and } \quad S(x)=2 x \quad \forall x .
$$

Then it can be verified that $f$ and $g$ are compatible but not conditionally compatible. 
Example 1.2 Let $X=[1,8]$ with the usual metric $d$. Define mappings $A, S: X \rightarrow X$ as follows:

$$
\begin{aligned}
& A(x)=2 \quad \text { if } x \leq 2, \quad A(x)=5 \quad \text { if } x>2, \\
& S(x)=6-2 x \quad \text { if } x \leq 2, \quad S(x)=8 \quad \text { if } x>2 .
\end{aligned}
$$

In this example $A$ and $S$ are conditionally compatible but not compatible. To see this, we can consider the constant sequence $z_{n}=2$, then $\lim _{n \rightarrow \infty} A\left(z_{n}\right)=2, \lim _{n \rightarrow \infty} S\left(z_{n}\right)=2$, $\lim _{n \rightarrow \infty} A\left(S\left(z_{n}\right)\right)=2, \lim _{n \rightarrow \infty} S\left(A\left(z_{n}\right)\right)=2$ and $\lim _{n \rightarrow \infty} d\left(A\left(S\left(z_{n}\right)\right), S\left(A\left(z_{n}\right)\right)\right)=0$. Again, if we consider the sequence $y_{n}=2-\frac{1}{n}$, then $\lim _{n \rightarrow \infty} A\left(y_{n}\right)=2, \lim _{n \rightarrow \infty} S\left(y_{n}\right)=\lim _{n \rightarrow \infty}(2+$ $\left.\frac{2}{n}\right) \rightarrow 2, \lim _{n \rightarrow \infty} A\left(S\left(y_{n}\right)\right)=5, \lim _{n \rightarrow \infty} S\left(A\left(y_{n}\right)\right)=2$ and $\lim _{n \rightarrow \infty} d\left(A\left(S\left(y_{n}\right)\right), S\left(A\left(y_{n}\right)\right)\right)=3$. Thus $f$ and $g$ are conditionally compatible but not compatible.

It may also be observed that conditional compatibility need not imply commutativity at the coincidence points. The following example illustrates this fact.

Example 1.3 Let $X=[0, \infty)$ and $d$ be the Euclidean metric $X$. Define $A$ and $S: X \rightarrow X$ by

$$
A(x)=x^{2} \quad \text { and } \quad S(x)= \begin{cases}x+6 & \text { if } x \in[0,9] \cup(16,+\infty) \\ x+72 & \text { if } x \in(9,16]\end{cases}
$$

In this example $A$ and $S$ are conditionally compatible, but they do not commute their only coincidence point $x=3$. To see this, let us consider the sequence $y_{n}=3+\frac{1}{n}$, then $\lim _{n \rightarrow \infty} A\left(y_{n}\right)=9=\lim _{n \rightarrow \infty} S\left(y_{n}\right)$ and $\lim _{n \rightarrow \infty} d\left(A\left(S\left(y_{n}\right)\right), S\left(A\left(y_{n}\right)\right)\right)=0$. Thus $f$ and $g$ are conditionally compatible. On the other hand, we have $A(x)=S(x)$ iff $x=3$ and $A(S(3))=$ $A(9)=81, S(A(3))=S(9)=15$. Then $A(3)=9=S(3)$, but $A(S(3)) \neq S(A(3))$.

In this paper we define the notion of conditionally compatible maps in a slightly different manner as follows.

Definition 1.2 Two self-mappings $A$ and $S$ of a metric space $(X, d)$ will be called to be faintly compatible iff $A$ and $S$ are conditionally compatible and $A$ and $S$ commute on a nonempty subset of coincidence points whenever the set of coincidences is nonempty.

If $A$ and $S$ are compatible, then they are obviously faintly compatible, but the converse is not true in general.

Example 1.4 Let $X=[3,6]$ and $d$ be the usual metric on $X$. Define self-mappings $A$ and $S$ on $X$ as follows:

$$
\begin{aligned}
& A(x)=3 \quad \text { if } x=3 \text { or } x>5, \quad A(x)=x+1 \quad \text { if } 3<x \leq 5, \\
& S(3)=3, \quad S(x)=\frac{x+7}{3} \quad \text { if } 3<x \leq 5, \quad S(x)=\frac{x+1}{2} \quad \text { if } x>5 .
\end{aligned}
$$

In this example $A$ and $S$ are faintly compatible but not compatible. To see this, if we consider the constant sequence $\left\{y_{n}=3\right\}$, then $A$ and $S$ are faintly compatible. On the other hand, if we choose the sequence $\left\{x_{n}=5+\frac{1}{n}\right\}$, then $\lim _{n \rightarrow \infty} A\left(x_{n}\right)=3=\lim _{n \rightarrow \infty} S\left(x_{n}\right)$, and 
$\lim _{n \rightarrow \infty} d\left(A\left(S\left(x_{n}\right)\right), S\left(A\left(x_{n}\right)\right)\right) \neq 0$. Thus $A$ and $S$ are faintly compatible, but they are not compatible.

It is also relevant to mention here that faint compatibility and noncompatibility are independent concepts. To see this, we can consider the following examples.

Example 1.5 Let $X=[2,10]$ and $d$ be the usual metric on $X$. Define self-mappings $A$ and $S$ on $X$ as follows:

$$
\begin{array}{ll}
A(x)=8 \quad \text { if } 2 \leq x \leq 5, \quad A(x)=2 \quad \text { if } x>5, \\
S(x)=2 \quad \text { if } 2 \leq x<5, \quad S(x)=x-3 \quad \text { if } x \geq 5 .
\end{array}
$$

In this example $A$ and $S$ are noncompatible, but not faintly compatible. To see this, let us consider the sequence $x_{n}=5+\frac{1}{n}$, then $\lim _{n \rightarrow \infty} A\left(x_{n}\right)=2, \lim _{n \rightarrow \infty} S\left(x_{n}\right)=\lim _{n \rightarrow \infty}=2$, but $\lim _{n \rightarrow \infty} d\left(A\left(S\left(x_{n}\right)\right), S\left(A\left(x_{n}\right)\right)\right)=6$. Thus $A$ and $S$ are noncompatible, but not faintly compatible.

Example 1.6 Let $X=[1, \infty)$ and let $d$ be the usual metric on $X$. Define $A, S: X \rightarrow X$ by

$$
A(x)=x^{2} \quad \forall x \quad \text { and } \quad S(x)=3 x-2 \quad \forall x .
$$

In this example $A$ and $S$ are faintly compatible, but not noncompatible.

Examples 1.5 and 1.6 clearly show that faint compatibility and noncompatibility are independent of each other.

If $A$ and $S$ are weakly compatible, then they are also faintly compatible, but the converse is not true in general (see examples on the following pages).

It is worth mentioning here that if $f$ and $g$ are owc, then they are also faintly compatible, but the converse is not true in general.

Example 1.7 Let $X=[1, \infty)$ and $d$ be the usual metric on $X$. Define $A, S: X \rightarrow X$ by

$$
A(x)=x \quad \forall x \quad \text { and } \quad S(x)=x+1 \quad \forall x .
$$

Then it can be verified that $A$ and $S$ are trivially faintly compatible but not owc.

It may be pointed out that the notion of owc implies commutativity at some coincidence points, but it does not help in establishing the existence of coincidence points, whereas the new notion is useful in establishing the existence of coincidence points.

\section{Main results}

Theorem 2.1 Let $A$ and $S$ be noncompatible faintly compatible self-mappings of a metric space $(X, d)$ satisfying

(i) $A X \subseteq S X$,

(ii) $d(A(x), A(y)) \leq k d(S(x), S(y)), 0 \leq k<1$.

If either $A$ or $S$ is continuous, then $A$ and $S$ have a unique common fixed point. 
Proof Noncompatibility of $A$ and $S$ implies that there exists some sequence $\left\{x_{n}\right\}$ in $X$ such that $A\left(x_{n}\right) \rightarrow t$ and $S\left(x_{n}\right) \rightarrow t$ for some $t \in X$, but $\lim _{n \rightarrow \infty} d\left(A\left(S\left(x_{n}\right)\right), S\left(A\left(x_{n}\right)\right)\right)$ is either non-zero or non-existent. Since $A$ and $S$ are faintly compatible and $\lim _{n \rightarrow \infty} A\left(x_{n}\right)=$ $\lim _{n \rightarrow \infty} S\left(x_{n}\right)=t$, there exists a sequence $\left\{z_{n}\right\}$ in $X$ satisfying $\lim _{n \rightarrow \infty} A\left(z_{n}\right)=\lim _{n \rightarrow \infty} S\left(z_{n}\right)=$ $u$ (say) such that $\lim _{n \rightarrow \infty} d\left(A\left(S\left(z_{n}\right)\right), S\left(A\left(z_{n}\right)\right)\right)=0$. Further, since $A$ is continuous, then $\lim _{n \rightarrow \infty} A\left(A\left(z_{n}\right)\right)=A(u)$ and $\lim _{n \rightarrow \infty} A\left(S\left(z_{n}\right)\right)=A(u)$. The last three limits together imply $\lim _{n \rightarrow \infty} S\left(A\left(z_{n}\right)\right)=A(u)$. Since $A X \subseteq S X$ implies that $A(u)=S(v)$ for some $v \in$ $X$ and $A\left(A\left(z_{n}\right)\right) \rightarrow S(v), S\left(A\left(z_{n}\right)\right) \rightarrow S(v)$. Also, using (ii), we get $d\left(A(v), A\left(A\left(z_{n}\right)\right)\right) \leq$ $k d\left(S(v), S\left(A\left(z_{n}\right)\right)\right)$. On letting $n \rightarrow \infty$, we get $A(v)=S(v)$. Thus $v$ is a coincidence point of $A$ and $S$. Further, faint compatibility implies $A(S(v))=S(A(v))$, and hence $A(S(v))=$ $S(A(v))=A(A(v))=S(S(v))$. If $A(v) \neq A(A(v))$, then using (ii) we get $d(A(v), A(A(v))) \leq$ $k d(S(v), S(A(v)))=k d(A(v), A(A(v)))$, a contradiction. Hence $A(v)$ is a common fixed point of $A$ and $S$. The same conclusion is obtained when $S$ is assumed to be continuous since the continuity of $S$ implies the continuity of $A$. The uniqueness of the common fixed point theorem is an easy consequence of the condition (ii).

We now give an example to illustrate Theorem 2.1.

Example 2.1 Let $X=[0,20]$ and let $d$ be the usual metric on $X$. Define $A, S: X \rightarrow X$ as follows:

$$
\begin{aligned}
& A x=\frac{x}{10} \text { if } x<10, A x=\frac{20-x}{10} \text { if } x \geq 10, \\
& S 0=0, S x=20-x \text { if } x>0 .
\end{aligned}
$$

Then $A$ and $S$ satisfy all the conditions of Theorem 2.1 and have a unique common fixed point at $x=0$. It can be verified in this example that $A$ and $S$ satisfy the condition (ii) with $k=\frac{1}{10}$. Furthermore, $A$ and $S$ are faintly compatible. Also, $A$ and $S$ are noncompatible. To see that, let us consider an increasing sequence $\left\{x_{n}\right\}$ in $X=[2,20]$ such that $x_{n} \rightarrow 20$. Then $A\left(x_{n}\right) \rightarrow 0, S\left(x_{n}\right) \rightarrow 0, A\left(S\left(x_{n}\right)\right) \rightarrow 0$ and $S\left(A\left(x_{n}\right)\right) \rightarrow 20$ as $n \rightarrow \infty$. Therefore, $A$ and $S$ are noncompatible.

It is well known that the strict contractive condition $d(A(x), A(y))<d(S(x), S(y))$ does not ensure the existence of common fixed points unless the space taken to be compact or some sequence of iterates is assumed to be a Cauchy sequence. The next theorem illustrates the applicability of faintly compatible mappings satisfying the strict contractive condition.

Theorem 2.2 Let $A$ and $S$ be noncompatible faintly compatible self-mappings of a metric space $(X, d)$ satisfying the condition (i) of Theorem 2.1 and

(i) $d(A(x), A(y))<d(S(x), S(y))$ whenever $S x \neq S y$.

If either $A$ or $S$ is continuous, then $A$ and $S$ have a unique common fixed point.

Proof Noncompatibility of $A$ and $S$ implies that there exists some sequence $\left\{x_{n}\right\}$ in $X$ such that $A\left(x_{n}\right) \rightarrow t$ and $S\left(x_{n}\right) \rightarrow t$ for some $t \in X$, but $\lim _{n \rightarrow \infty} d\left(A\left(S\left(x_{n}\right)\right), S\left(A\left(x_{n}\right)\right)\right) \neq 0$ or nonexistent. Since $A$ and $S$ are faintly compatible, there exists a sequence $\left\{z_{n}\right\}$ in $X$ satisfying $\lim _{n \rightarrow \infty} A\left(z_{n}\right)=\lim _{n \rightarrow \infty} S\left(z_{n}\right)=v$ (say) such that $\lim _{n \rightarrow \infty} d\left(A\left(S\left(z_{n}\right)\right), S\left(A\left(z_{n}\right)\right)\right)=0$. Further, since $A$ is continuous, then $\lim _{n \rightarrow \infty} A\left(A\left(z_{n}\right)\right)=A(v)$ and $\lim _{n \rightarrow \infty} A\left(S\left(z_{n}\right)\right)=A(v)$. The last three limits together imply $\lim _{n \rightarrow \infty} S\left(A\left(z_{n}\right)\right)=A(v)$. Since $A X \subseteq S X$ implies that $A(v)=S(w)$ for some $w \in X$ and $A\left(A\left(z_{n}\right)\right) \rightarrow S(w), S\left(A\left(z_{n}\right)\right) \rightarrow S(w)$. Also, using (i) we get $d\left(A(w), A\left(A\left(z_{n}\right)\right)\right)<d\left(S(w), S\left(A\left(z_{n}\right)\right)\right)$. On letting $n \rightarrow \infty$, we get $A(w)=S(w)$. Again, in 
view of faint compatibility of $A$ and $S$, we get $A(S(w))=S(A(w))$, and hence $A(S(w))=$ $S(A(w))=A(A(w))=S(S(w))$. We claim that $A(w)=A(A(w))$. If not, by virtue of (i), we get

$$
d(A(w), A(A(w)))<d(S(w), S(A(w)))=d(A(w), A(A(w))),
$$

which gives a contradiction implying thereby $A(w)=A(A(w))=S(A(w))$. Hence, $A w$ is a common fixed point of $A$ and $S$. The same conclusion can be drawn when $S$ is assumed to be continuous since the continuity of $S$ implies the continuity of $A$. The uniqueness of the common fixed point follows from (i).

Remark 2.1 We have proved Theorem 2.2 under the simplest contractive condition $d(A(x), A(y))<d(S(x), S(y))$. Analogous results for commuting or compatible mappings require a Banach-type or $d(A(x), A(y)) \leq \phi(d(S(x), S(y)))$ or Meir-Keeler-type contractive condition [19].

We now show that the notion of faint compatibility is also useful in studying fixed points of mappings satisfying Lipschitz-type conditions.

Theorem 2.3 Let $A$ and $S$ be noncompatible faintly compatible self-mappings of a metric space $(X, d)$ satisfying the condition (i) of Theorem 2.1 and

(i) $d(A(x), A(y)) \leq k d(S(x), S(y)), k \geq 0$;

(ii) $d(A(x), A(A(x))) \neq \max \{d(A(x), S(A(x))), d(S(A(x)), A(A(x)))\}$ whenever the right-hand side is non-zero.

Suppose either $A$ or $S$ is continuous, then $A$ and $S$ have a common fixed point.

Proof Noncompatibility of $A$ and $S$ implies that there exists some sequence $\left\{x_{n}\right\}$ in $X$ such that $A\left(x_{n}\right) \rightarrow t$ and $S\left(x_{n}\right) \rightarrow t$ for some $t \in X$, but $\lim _{n \rightarrow \infty} d\left(A\left(S\left(x_{n}\right)\right), S\left(A\left(x_{n}\right)\right)\right) \neq 0$ or nonexistent. Since $A$ and $S$ are faintly compatible and $\lim _{n \rightarrow \infty} A\left(x_{n}\right)=\lim _{n \rightarrow \infty} S\left(x_{n}\right)=t$, there exists a sequence $\left\{z_{n}\right\}$ in $X$ satisfying $\lim _{n \rightarrow \infty} A\left(z_{n}\right)=\lim _{n \rightarrow \infty} S\left(z_{n}\right)=v$ (say) such that $\lim _{n \rightarrow \infty} d\left(A\left(S\left(z_{n}\right)\right), S\left(X\left(z_{n}\right)\right)\right)=0$. Further, since $A$ is continuous, then $\lim _{n \rightarrow \infty} A\left(A\left(z_{n}\right)\right)=$ $A(v)$ and $\lim _{n \rightarrow \infty} A\left(S\left(z_{n}\right)\right)=A(v)$. The last three limits together imply $\lim _{n \rightarrow \infty} S\left(A\left(z_{n}\right)\right)=$ $A(v)$. Since $A X \subseteq S X$ implies that $A(v)=S(w)$ for some $w \in X$ and $A\left(A\left(z_{n}\right)\right) \rightarrow S(w)$, $S\left(A\left(z_{n}\right)\right) \rightarrow S(w)$. Also, using (i), we get $d\left(A(w), A\left(A\left(z_{n}\right)\right)\right) \leq k d\left(S(w), S\left(A\left(z_{n}\right)\right)\right)$. On letting $n \rightarrow \infty$, we get $A(w)=S(w)$. This implies that $w$ is a coincidence point of $A$ and $S$. In view of faint compatibility of $A$ and $S$, we get $A(S(w))=S(A(w))=A(A(w))=S(S(w))$. We claim that $A(w)=A(A(w))$. If not, by virtue of (ii), we get

$$
\begin{aligned}
d(A(w), A(A(w))) & \neq \max \{d(A(w), S(A(w))), d(A(A(w)), S(A(w)))\} \\
& =d(A(w), A(A(w))),
\end{aligned}
$$

which gives a contradiction. Hence $A(w)=A(A(w))=A(S(w))$ and $A w$ is a common fixed point of $A$ and $S$. The same conclusion is obtained when $S$ is assumed to be continuous since the continuity of $S$ implies the continuity of $A$. This completes the proof.

We now give an example [20] to illustrate Theorem 2.3. 
Example 2.2 Let $X=[0,1]$ with the Euclidean metric $d$. Define mappings $A, S: X \rightarrow X$ by

$$
A(x)=\frac{1}{2}-\left|\frac{1}{2}-x\right| \text { and } S(x)=\frac{2(1-x)}{3} .
$$

Then $A$ and $S$ satisfy all the conditions of Theorem 2.3 and have a common fixed point $x=\frac{2}{5}$ and a coincidence point $x=1$ at which $A$ and $S$ do not commute. It may be verified in this example that $A$ and $S$ satisfy the condition (i) for $k=\frac{3}{2}$ together with the condition (ii). The mappings $A$ and $S$ are faintly compatible (take a constant sequence $y_{n}=\frac{2}{5}$ ) and they commute at the coincidence point at $x=\frac{2}{5}$. Moreover, $A$ and $S$ are noncompatible (consider a sequence $x_{n}=1-\frac{1}{n}$ ).

In Example 2.2, $A$ and $S$ do not commute at the coincidence point $x=1$, hence they do not satisfy the condition of weakly compatible mappings.

The next example also illustrates Theorem 2.3.

Example 2.3 Let $X=[0,1]$ and let $d$ be the usual metric on $X$. Define self-mappings $A$ on $X$ as in the above Example 2.1 and $S: X \rightarrow X$ by

$$
S(x)=\frac{2}{3} \cdot \text { fractional part of }(1-x)
$$

Then $A$ and $S$ satisfy all the conditions of Theorem 2.3 and have two common fixed points $x=\frac{2}{5}$ and $x=1$.

As an application of faint compatible mappings, we now prove a common fixed point theorem under a more general condition that may hold for mappings satisfying contractive as well as nonexpansive and Lipschitz-type conditions.

Theorem 2.4 Let $A$ and $S$ be noncompatible faintly compatible self-mappings of a metric space $(X, d)$ satisfying

(i) $d(A(x), A(A(x))) \neq \max \{d(A(x), S(A(x))), d(S(A(x)), A(A(x)))\}$ whenever the right-hand side is non-zero.

Suppose $A$ and $S$ are continuous, then $A$ and $S$ have a common fixed point.

Proof Noncompatibility of $A$ and $S$ implies that there exists some sequence $\left\{x_{n}\right\}$ in $X$ such that $A\left(x_{n}\right) \rightarrow t$ and $S\left(x_{n}\right) \rightarrow t$ for some $t \in X$, but $\lim _{n \rightarrow \infty} d\left(A\left(S\left(x_{n}\right)\right), S\left(A\left(x_{n}\right)\right)\right) \neq$ 0 or nonexistent. The continuity of $A$ and $S$ implies that $\lim _{n \rightarrow \infty} A\left(S\left(x_{n}\right)\right)=A(t)$, and $\lim _{n \rightarrow \infty} S\left(A\left(x_{n}\right)\right)=S(t)$. In view of faint compatibility and continuity of $A$ and $S$, we can easily obtain a common fixed point as has been proved in the corresponding part of Theorem 2.3.

Examples 2.2 and 2.3 also illustrate Theorem 2.4.

Remark 2.2 It may be in order to point out here that Theorems 2.1, 2.2, 2.3 and 2.4 have been proved under a noncomplete metric space. Further, it would be interesting to apply the technique of Haghi et al. [11] in the above results. 
Remark 2.3 Theorem 2.4 remains true if one replaces the condition (i) by any one of the following conditions (see also [21]):

(a) $d(S(x), S(S(x))) \neq \max \{d(S(x), A(S(x))), d(A(S(x)), S(S(x)))\}$,

(b) $d(A(x), A(A(x))) \neq d(A(x), S(x))+d(S(x), A(A(x)))$,

(c) $d(S(x), S(S(x))) \neq d(S(x), A(x))+d(A(x), S(S(x)))$ whenever the right-hand side is non-zero.

Remark 2.4 Faint compatibility is a necessary condition for the existence of common fixed points of given mappings $A$ and $S$ satisfying contractive or more general Lipschitz-type mapping pairs. Let $A$ and $S$ be a Lipschitz-type pair of self-mappings of a metric space $(X, d)$ and let $A$ and $S$ have a common fixed point $x$. Then $A(x)=$ $S(x)=x$ and $A(S(x))=S(A(x))=A(x)=S(x)=x$. If we choose the constant sequence $x_{n}=x$, then $\lim _{n \rightarrow \infty} A\left(x_{n}\right)=\lim _{n \rightarrow \infty} S\left(x_{n}\right)=\lim _{n \rightarrow \infty} A\left(S\left(x_{n}\right)\right)=\lim _{n \rightarrow \infty} S\left(A\left(x_{n}\right)\right)=x$ and $\lim _{n \rightarrow \infty} d\left(A\left(S\left(x_{n}\right)\right), S\left(A\left(x_{n}\right)\right)\right)=d(x, x)=0$, that is, $A$ and $S$ are faintly compatible.

\section{Competing interests}

The authors declare that they have no competing interests.

\section{Authors' contributions}

All authors read and approved the final manuscript.

\section{Author details}

'Department of Mathematics-Applied Sciences and Humanities, Bipin Tripahti Kumaon Institute of Technology, Uttarakhand Technical University, Almora, Dwarahat, 262553, India. ${ }^{2}$ Department of Mathematics, King Abdulaziz University, P.O. Box 80203, Jeddah, 21589, Saudi Arabia.

\section{Acknowledgements}

The authors are thankful to the referees for their valuable suggestions for improving the presentation of the paper. The research of N. Shahzad was partially supported by the Deanship of Scientific Research (DSR), King Abdulaziz University, Jeddah, Saudi Arabia.

Received: 18 January 2013 Accepted: 15 April 2013 Published: 18 June 2013

\section{References}

1. Boyce, WB: Commuting functions with common fixed point. Trans. Am. Math. Soc. 137, 77-92 (1969)

2. Huneke, JP: On common fixed points of commuting continuous functions on an interval. Trans. Am. Math. Soc. 139, 371-381 (1969)

3. Sessa, S: On a weak commutativity condition of mappings in fixed point considerations. Publ. Inst. Math. 32, 149-153 (1982)

4. Jungck, G: Common fixed points for noncontinuous nonself maps on nonmetric spaces. Far East J. Math. Sci. 4 199-215 (1996)

5. Pant, RP: Common fixed points of noncommuting mappings. J. Math. Anal. Appl. 188, 436-440 (1994)

6. Al-Thagafi, MA, Shahzad, N: Generalized /-nonexpansive selfmaps and invariant approximations. Acta Math. Sin. 24, 867-876 (2008)

7. Pant, V, Pant, RP: Common fixed points of conditionally commuting maps. Fixed Point Theory 1, 113-118 (2010)

8. Alghamdi, MA, Radenović, S, Shahzad, N: On some generalizations of commuting mappings. Abstr. Appl. Anal. 2012, Article ID 952052 (2012)

9. Đorić, D, Kadelburg, Z, Radenović, S: A note on occasionally weakly compatible and common fixed points. Fixed Point Theory 13, 475-480 (2012)

10. Pant, RP, Bisht, RK: Occasionally weakly compatible mappings and fixed points. Bull. Belg. Math. Soc. Simon Stevin 19, 655-661 (2012)

11. Haghi, RH, Rezapour, S, Shahzad, N: Some fixed point generalizations are not real generalizations. Nonlinear Anal. 74, 1799-1803 (2011)

12. Ćirić, L, Samet, B, Vetro, C: Common fixed point theorems for families of occasionally weakly compatible mappings. Math. Comput. Model. 53(5-6), 631-636 (2011)

13. Ćirić, L, Samet, B, Cakić, N, Damjanović, B: Generalized $(\psi, \omega)$-weak nonlinear contractions in ordered K-metric spaces. Comput. Math. Appl. 62, 3305-3316 (2011)

14. Cirić, L, Razani, A, Radenović, S, Ume, JS: Common fixed point theorems for families of weakly compatible maps. Comput. Math. Appl. 55(11), 2533-2543 (2008)

15. Ćirić, LB, Nikolić, NT, Ume, JS: Common fixed point theorems for weakly compatible quasi contraction mappings. Acta Math. Hung. 113(4), 257-267 (2006) 
16. Abbas, M, Ćirić, L, Damjanović, B, Khan, MA: Coupled coincidence and common fixed point theorems for hybrid pair of mappings. Fixed Point Theory Appl. (2012). doi:10.1186/1687-1812-2012-4

17. Jungck, G: Compatible mappings and common fixed points. Int. J. Math. Math. Sci. 9(4), 771-779 (1986)

18. Bouhadjera, H, Godet-Thobie, C: Common fixed point theorems for pair of subcompatible maps. arXiv:0906.3159v1 [math. FA] (2009)

19. Meir, A, Keeler, E: A theorem on contraction mappings. J. Math. Anal. Appl. 28, 326-329 (1969)

20. Bisht, RK: Common fixed points of generalized Meir-Keeler type condition and nonexpansive mappings. Int. J. Math. Math. Sci. 2012, Article ID 786814 (2012)

21. Pant, RP: Common fixed points of Lipschitz type mapping pairs. J. Math. Anal. Appl. 240, 280-283 (1999)

doi:10.1186/1687-1812-2013-156

Cite this article as: Bisht and Shahzad: Faintly compatible mappings and common fixed points. Fixed Point Theory and Applications 2013 2013:156.

\section{Submit your manuscript to a SpringerOpen ${ }^{\circ}$ journal and benefit from:}

- Convenient online submission

- Rigorous peer review

Immediate publication on acceptance

- Open access: articles freely available online

- High visibility within the field

- Retaining the copyright to your article 